\title{
Multiple Sclerosis: Neurofilament Pathology in Spinal Motor Neurons
}

\section{Kathrin S Muller-Wielsch ${ }^{1,2 *}$, Barbara Cannella' and Cedric S Raine ${ }^{1}$}

${ }^{1}$ Department of Pathology (Neuropathology), Albert Einstein College of Medicine, 1300 Morris Park Avenue, Bronx, NY 10461, USA

${ }^{2}$ Charité University Medicine Berlin, Charitéplatz 1, 10117 Berlin, Germany

\begin{abstract}
Objective: While traditionally a disorder of myelin, in multiple sclerosis (MS) neuronal and axonal damage has in recent years become an important topic of clinical relevance. To address this, alterations in neurofilament phosphorylation, known markers of neuronal health, were investigated in anterior horn cells in MS spinal cord tissue for signs of motor neuron damage.
\end{abstract}

Methods: Spinal cord tissue was examined from 13 MS and 6 control patients. Fresh frozen sections were labelled with antibodies against phosphorylated and non-phosphorylated epitopes of neurofilament $\mathrm{H}(\mathrm{NF}-\mathrm{H})$ and analyzed by light microscopy.

Results: In MS, increased expression of phosphorylated NF-H in spinal motor neuron perikarya (abnormal for neurons) occurred in $61.5 \%$ of cases, mostly in chronic active lesions, with the strongest immunoreactivity at the lumbar level. Inflammatory activity was common in chronic active but rare in chronic silent lesions. In one case with an acute lesion, we saw swollen axons positive for non-phosphorylated NF-H, a pathologic marker in axons, but no signs of perikaryal damage. Expression of non-phosphorylated NF-H in spinal motor neuron perikarya was similar in both MS and controls.

Conclusion: In line with previous studies, our findings implicate anterior horn cell damage as a common feature in MS. We propose that underlying mechanisms may involve reduced synaptic input and/or retrograde degeneration, subjects which remain to be investigated

Keywords: Multiple sclerosis; Neuronal damage; Anterior horn cells; Spinal cord; Neurofilaments

\section{Introduction}

Multiple sclerosis (MS) is the leading cause of non-traumatic neurologic disability among young adults in Europe and North America [1]. A major component of its pathology is destruction of myelin and oligodendrocytes against a background of inflammation, sometimes leading to reversible neurological dysfunction. However, irreversible disability in MS is usually attributed to neuronal damage $[2,3]$, the reason for the recent focus of attention on this topic in MS research. Axonal damage [2-5], neuronal apoptosis [6,7] and neuronal loss [8] in grey and white matter lesions have been described both in MS and its widely-used animal model, experimental autoimmune encephalomyelitis (EAE). In spinal cord, substantially reduced nerve cell counts were found both in EAE [9] and MS [10-12].

In the search for molecular markers of neuronal pathology, neurofilament phosphorylation has proven useful. Neurofilaments (NF) are a major structural component of the neuronal cytoskeleton, particularly prominent in large neurons with long axons [13]. In healthy neurons, neurofilaments in perikarya are non-phosphorylated in contrast to those of axons in which neurofilaments are phosphorylated [14]. Thus, phosphorylated NF in damaged neuronal perikarya and non-phosphorylated NF in injured axons are well established indicators of pathology $[15,16]$.

The phenomenon of neuronal NF phosphorylation has been used to visualize axonal damage in the MS brain [4], as well as axonal and motor neuron perikaryal damage in EAE spinal cord $[17,18]$. Interestingly, recent reports on the spinal cord in MS that examined NF phosphorylation in spinal cord neurons, emphasized axonal changes $[12,19]$. In the present study on spinal cord in MS, we have investigated NF phosphorylation in perikarya of anterior horn cells and were impressed by the frequency of anterior horn cell (motor neuron) anomalies in active lesions and the degree of neuronal loss at later stages.

\section{Material and Methods}

\section{Tissue material}

We examined early autopsy, fresh frozen spinal cord tissue from 13 MS patients ( 9 female, 4 male, mean age $48.3 \pm 11.6$ years) and 5 control cases ( 2 male with other neurological diseases (OND), mean age $52.5 \pm 21.5$ years; 1 female, 2 male with non-neurological diseases, mean age $67.0 \pm 28.7$ years) (Table 1). MS lesions were classified in acute, chronic active or chronic silent according to standard criteria [20]. Briefly, "acute" refers to fresh white matter lesions with parenchymal and perivascular collections of infiltrating cells, edema, ongoing myelin and axonal damage, astroglial hypertrophy, microglial reactivity and an indistinct margin; "chronic silent" covers total loss of myelin, depletion of axons, scarring fibrous astrogliosis, microglial reactivity and a sharp edge to the lesion; "chronic active" lesions are mostly areas of damaged white matter showing glial scarring, myelin loss and axonal depletion with a sharp edge along which more recent activity consisting of inflammatory changes and myelin loss, is seen. Spinal cords were obtained with a post-mortem interval of $9.0 \pm 5.8$ $\mathrm{h}$ in MS patients and $4.2 \pm 1.2 \mathrm{~h}$ in control cases. Segments of fresh spinal cord $0.5-1 \mathrm{~cm}$ in length were embedded end-on in Tissue-Tek O.C.T. Compound (Sakura Finetek, Torrance, CA, USA), snap-frozen on dry ice and kept at $-80^{\circ} \mathrm{C}$ until use. Transverse sections $10 \mu \mathrm{m}$ thick were cut on a Cryostat (Thermo Electron Corporation, Pittsburgh, PA,

*Corresponding author: Muller-Wielsch KS, Department of Pathology (Neuropathology), Albert Einstein College of Medicine, 1300 Morris Park Avenue Bronx, NY 10461, USA, Tel: +1-(718)-430-2495; Fax: +1-(718)-678-1018 Email: cedric.raine@einstein.yu.edu

Received June 14, 2017; Accepted August 04, 2017 ; Published August 11, 2017

Citation: Muller-Wielsch KS, Cannella B, Raine CS (2017) Multiple Sclerosis: Neurofilament Pathology in Spinal Motor Neurons. J Mult Scler (Foster City) 4 207. doi: 10.4172/2376-0389.1000207

Copyright: () 2017 Muller-Wielsch KS, et al. This is an open-access article distributed under the terms of the Creative Commons Attribution License, which permits unrestricted use, distribution, and reproduction in any medium, provided the original author and source are credited 


\begin{tabular}{|c|c|c|c|c|c|c|c|}
\hline Code & Age & Sex & Diagnosis & Lesion Stage & Cause of Death & Post Mortem Interval (hrs) & Spinal Cord Level \\
\hline MS1 & 31 & $f$ & Chronic Prog. MS & Acute & Respiratory Failure & 3 & $\mathrm{C}$ \\
\hline MS2 & 45 & $f$ & MS & Chronic active & Respiratory failure & 5 & L \\
\hline MS3 & 32 & $f$ & Chronic Prog. MS & Chronic active & Bronchopneumonia & 12 & $\mathrm{C}$ \\
\hline MS4 & 27 & $f$ & Chronic Prog. MS & Chronic active & Sepsis & 4 & Th \\
\hline MS5 & 56 & $f$ & Chronic Prog. MS & Chronic silent & Sepsis, Pneumonia & 2 & L \\
\hline MS6 & 55 & $f$ & Chronic Prog. MS & Chronic silent & Sepsis & 8 & L \\
\hline MS7 & 46 & $\mathrm{~m}$ & Chronic Prog. MS & Chronic silent & Cardiac arrest & 8 & L \\
\hline MS8 & 69 & $\mathrm{~m}$ & Chronic Prog. MS & Chronic silent & Bronchopneumonia, Adeno-Ca & 6 & Th \\
\hline MS9 & 72 & $\mathrm{~m}$ & Chronic Prog. MS & Chronic silent & Colon-Ca & 8 & $\mathrm{C}$ \\
\hline MS10 & 36 & $f$ & Chronic Prog. MS & Chronic silent & Pneumonia & 8 & L \\
\hline MS11 & 46 & $\mathrm{~m}$ & Chronic Prog. MS & Chronic silent & Bronchopneumonia & 16 & $\mathrm{C}$ \\
\hline MS12 & 54 & $f$ & Chronic Prog. MS & Chronic silent & Bronchopneumonia & 24 & $\mathrm{~L}(+\mathrm{Th})$ \\
\hline MS13 & 59 & $f$ & MS & Chronic silent & Bronchopneumonia & 12 & L \\
\hline OND1 & 74 & $\mathrm{~m}$ & Polyglucosan Inclusion Disease & $\mathrm{n} / \mathrm{a}$ & Urosepsis & 5 & Th \\
\hline OND2 & 31 & $\mathrm{~m}$ & $\begin{array}{l}\text { Olivopontocerebellar } \\
\text { Degeneration }\end{array}$ & $\mathrm{n} / \mathrm{a}$ & Bronchopneumonia & 4 & $\mathrm{C}$ \\
\hline $\mathrm{HC} 1$ & 81 & $\mathrm{~m}$ & Normal CNS & $\mathrm{n} / \mathrm{a}$ & Myocardial infarct & 3 & Th \\
\hline $\mathrm{HC} 2$ & 86 & $f$ & Normal CNS & $\mathrm{n} / \mathrm{a}$ & Pulmonary embolus & 6 & Th \\
\hline $\mathrm{HC} 3$ & 34 & $\mathrm{~m}$ & Normal CNS & $\mathrm{n} / \mathrm{a}$ & Leukemia & 3 & C \\
\hline
\end{tabular}

Table 1: Patient population.

USA) and stored on glass slides at $-20^{\circ} \mathrm{C}$ until use. The procedure for obtaining and processing human tissue was approved according to an Institutional Review Board protocol (IRB number 89-31) (Table 1).

\section{Immunohistochemistry}

As primary antibodies, we used SMI-31, a mouse monoclonal immunoglobulin G (IgG) binding a phosphorylated epitope of neurofilament $\mathrm{H}$, abbreviated in the figures as "P-NF-H" (BioLegend, San Diego, CA, USA), and SMI-32, a mouse monoclonal IgG binding a non-phosphorylated epitope of neurofilament $\mathrm{H}$, abbreviated in the figures as "Non-P-NF-H" (BioLegend), both diluted 1:1000 in Tris buffered saline containing 0.1\% Tween 20 (TBS/Tween). Biotinylated goat anti-mouse IgG, diluted 1:300 was used as secondary antibody (Vector Laboratories, Burlingame, CA, USA). Binding was revealed by an avidin-biotin-peroxidase method (Vectastain ABC solution, Vector Laboratories, 1:100), and visualized with 3,3' -diaminobenzidine (DAB) solution containing $0.5 \mathrm{mg} / \mathrm{ml} \mathrm{DAB}$ and $0.1 \%$ hydrogen peroxide in TBS.

Sections were dried at room temperature for $30 \mathrm{~min}$, then fixed in $2 \%$ paraformaldehyde in phosphate buffered saline (PBS) for $5 \mathrm{~min}$ and washed 3 times, 5 min each, in TBS/Tween. Endogenous peroxidase activity was blocked by incubation with $0.1 \%$ hydrogen peroxide for $30 \mathrm{~min}$. Sections were washed twice and incubated in 10\% normal goat serum (NGS). After $30 \mathrm{~min}$, excess serum was removed and sections were incubated with primary antibody (1:1000 in 2\% NGS in TBS/ Tween), overnight at $4^{\circ} \mathrm{C}$. Sections were washed twice, incubated with biotinylated goat anti-mouse IgG (1:300 in 2\% NGS) for $90 \mathrm{~min}$ and then after 3 washes incubated in ABC solution (1:100) for $90 \mathrm{~min}$. Sections were then washed and incubated with DAB solution $(0.5 \mathrm{mg} /$ $\mathrm{ml}$ ) in TBS containing $0.1 \%$ hydrogen peroxide for $10 \mathrm{~min}$ to visualize reaction product. Negative controls omitting the primary antibody or using an irrelevant antibody were used. Sections were washed in distilled $\mathrm{H}_{2} \mathrm{O}$, dehydrated in ethanol and xylene and cover slipped.

\section{Section Analysis}

Hematoxylin and eosin-stained and immuno-stained sections were analyzed by light microscopy (Axioskop, Carl Zeiss, Thornwood, NY, USA). We concentrated on perikarya in lamina IX, the area where anterior horn cells are situated in the spinal cord. Cases showing positive immunostaining for SMI-31, i.e., abnormally phosphorylated NF-H, in neuronal perikarya were counted as positive. Cases not showing this reaction were counted as negative.

For the neuron count of a subgroup of MS cases, 5 serial sections of each patient stained with Nissl stain $(0.075 \%$ cresyl violet) were examined with stereological methods using StereoInvestigator software (MicroBrightField Inc., Williston, VT, USA). In the anterior horn as the region of interest, all neuronal perikarya were counted, measured and grouped according to their size into motor neurons (diameter $\geq 24 \mu \mathrm{m}$ ) and interneurons (diameter $(\geq 10-24 \mu \mathrm{m})$ [21]. For evaluation, total numbers of motor neurons per patient were compared. Further details of the procedure can be found in an earlier publication [11].

Images were captured with a digital camera (Axiocam and software by Carl Zeiss); GraphPadPrism software was used for graph production.

\section{Results}

Established, gliotic lesions devoid of myelin and displaying variable degrees of axonal degeneration were common in all cases with chronic lesions but inflammatory changes (reflecting ongoing demyelination), were only seen in chronic active lesions. Such changes comprised perivascular cuffs and scattered lipid-laden macrophages in the white matter (mainly anterior or lateral columns) and occasional collections of small lymphocytes within the meninges.

By immunocytochemistry, clear differences were seen between MS and control cases in the expression of phosphorylated NF-H in anterior horn cell perikarya. Specifically, eight of thirteen MS patients showed widespread phosphorylated (abnormal) NF-H positivity compared to none of the controls (3 healthy, 2 OND: olivopontocerebellar degeneration and polyglucosan inclusion disease). Expression of nonphosphorylated (normal) NF-H in anterior horn cell perikarya was positive in all MS cases except one and in all controls (Figures 1a and 2).

Phosphorylated NF-H (abnormal) immunoreactivity of anterior horn cell perikarya was seen in all three cases with chronic active lesions regardless of spinal cord level, but in only five of nine of cases with chronic silent lesions, and none was seen in the single 


\section{a. Neurofilament phosphorylation in spinal motor neuron perikarya}

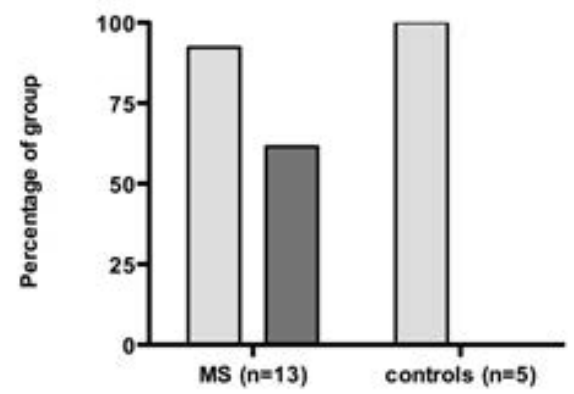

non-phosphorylated NF.H

\section{b. Pathologic phosphorylation in perikarya depending on lesion type}

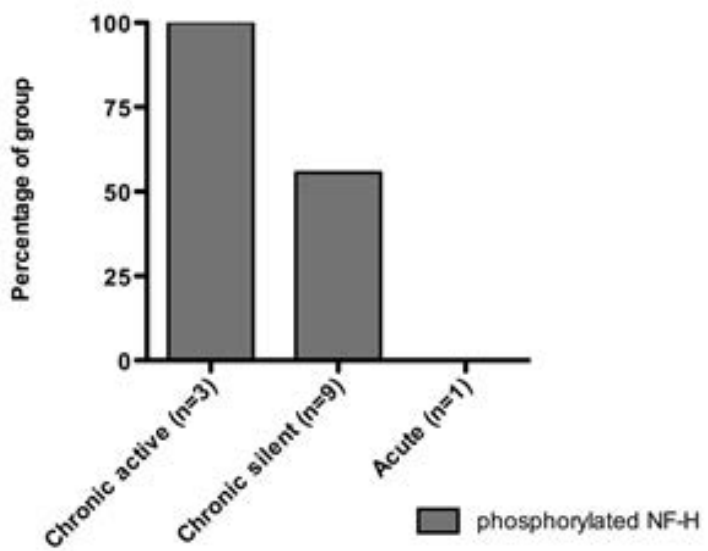

Figure 1: Neurofilament phosphorylation in spinal motor neuron perikarya in MS patients (left) and controls (right). Note that in MS patients there are abnormally phosphorylated NF-H in approximately two thirds of the group compared to none in the control group. Pathologic phosphorylation in perikarya depending on lesion type: chronic active (left), chronic silent (middle) and acute (right).

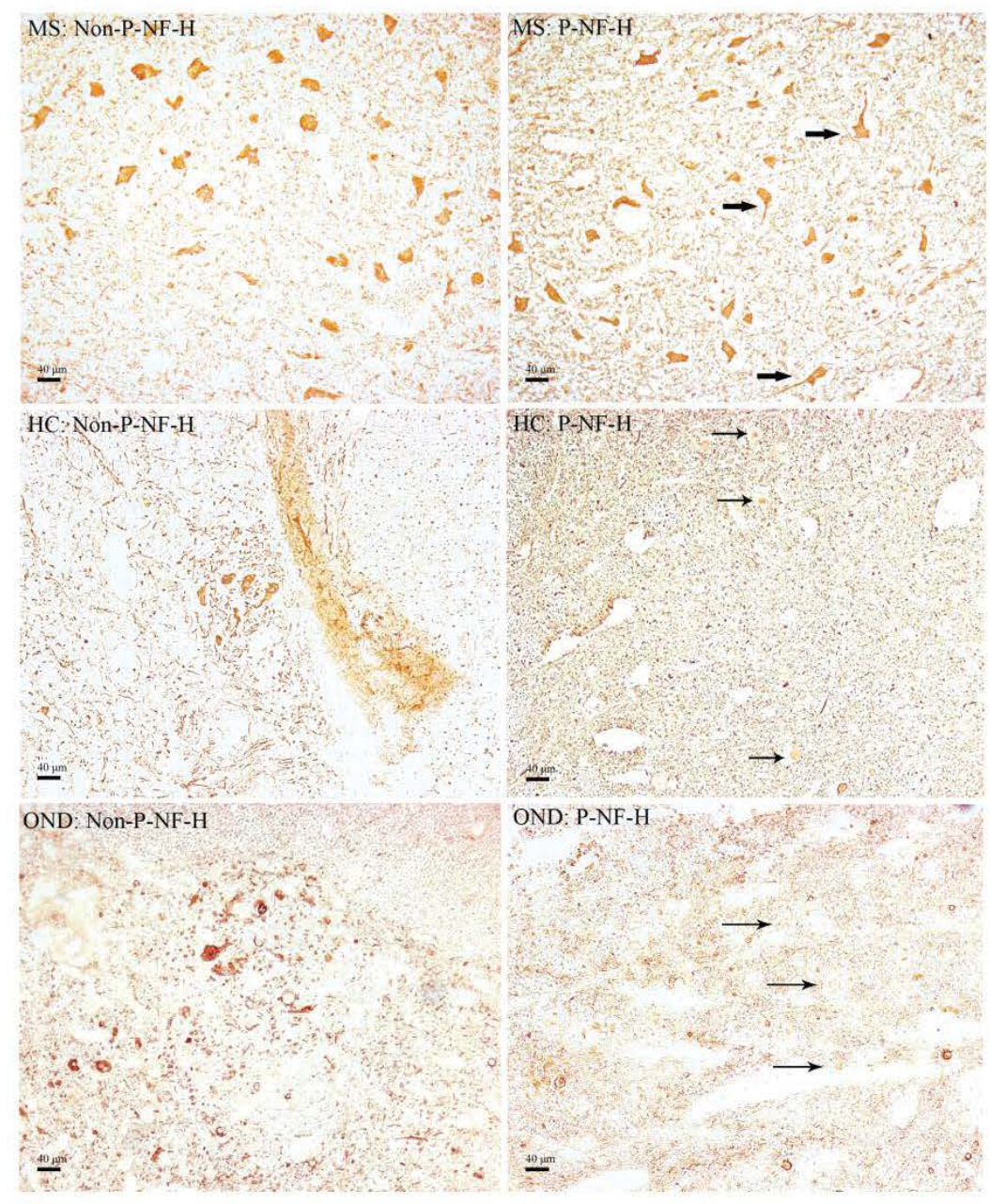

Figure 2: Spinal cord sections displaying immunoreactivity in perikarya against normal, non-phosphorylated epitopes of neurofilament $\mathrm{H}$ (Non-P-NF-H, left column) and abnormal, phosphorylated epitopes of neurofilament $\mathrm{H}$ (P-NF-H, right column). In the upper row are two images of a case of MS; The middle row, images for a neurologically healthy control (HC), and in the lower row, images of a case of polyglucosan inclusion disease, an OND control. Note that anterior horn cell perikarya contain prominent expression of phosphorylated NF-H (thick arrows) only in MS, while in both controls; perikarya remain non-reactive (thin arrows). 
case with an acute lesion examined (Figure 1b). Perikarya expressing phosphorylated NF-H were found at all levels of spinal cord. The highest intensity was found at lumbar levels. At thoracic and cervical levels, immunoreactivity was less pronounced. This pattern was particularly evident within one individual case where sections of multiple levels of spinal cord were available (Figure 3). In a subgroup of 4 lumbar patients, the neuron count was compared between cases showing abnormal NF-H phosphorylation in perikarya (positive) and those that did not (negative). In this quantitative comparison, the negative case had a neuron count of only $72,1 \%$ of the mean neuron count of the positive cases (figure not shown).

In the one case with an acute lesion, in which spinal cord sections showed scattered perivascular cuffs of lymphocytes in the white matter and an abundance of lipid-laden macrophages, axons with increased diameter were common, indicative of incipient degeneration. Some of these swollen axons expressed non-phosphorylated NF-H, consistent with ongoing damage (Figure 4). Neurofilaments in perikarya in this acute lesion were normal and non-phosphorylated. None of the other MS cases (with chronic active or silent lesions), displayed overt evidence of ongoing axonal damage such as swellings, spheroids or manifestations of neurofilament pathology.

\section{Discussion}

In this short report, we have documented high levels of phosphorylated NF-H immunoreactivity in anterior horn cell perikarya in fresh frozen, early autopsy spinal cord tissue from a group of 13 cases of MS. About two thirds of the group displayed this phenomenon. Similar changes have been reported previously in spinal motor neuron perikarya in amyotrophic lateral sclerosis (ALS), where damage to lower motor neurons is a characteristic feature [22]. In our study, we also examined a case of ALS as a positive control, which indeed showed phosphorylated NF-H immunoreactivity in perikarya (not shown). In other OND, in which spinal motor neurons are spared, one might not anticipate such changes. Accordingly, our OND control cases of olivopontocerebellar degeneration and polyglucosan inclusion disease did not show increased phosphorylation of NF-H in anterior horn cell perikarya, nor did neurologically healthy controls. Thus, we conclude that damage to spinal motor neuron perikarya indicated by abnormally increased NF phosphorylation is not a rare but rather a common feature of MS pathology.

In our single case with an acute MS lesion, we found pathologic NF-H only in axons, but not in perikarya. This might indicate that in early stages of lesion formation, axonal degeneration precedes motor neuron perikaryal damage. Correspondingly, Bannerman et al. found in mice on day 21 post-sensitization for EAE a large number of disrupted axons of which a significant majority displayed pathologic NF, but only the occasional anterior horn cell was positive for abnormal (phosphorylated) NF-H [18].

Of the cases with chronic active lesions, all samples revealed phosphorylated (pathologic) NF in perikarya of anterior horn cells compared to a little more than half of the cases with chronic silent
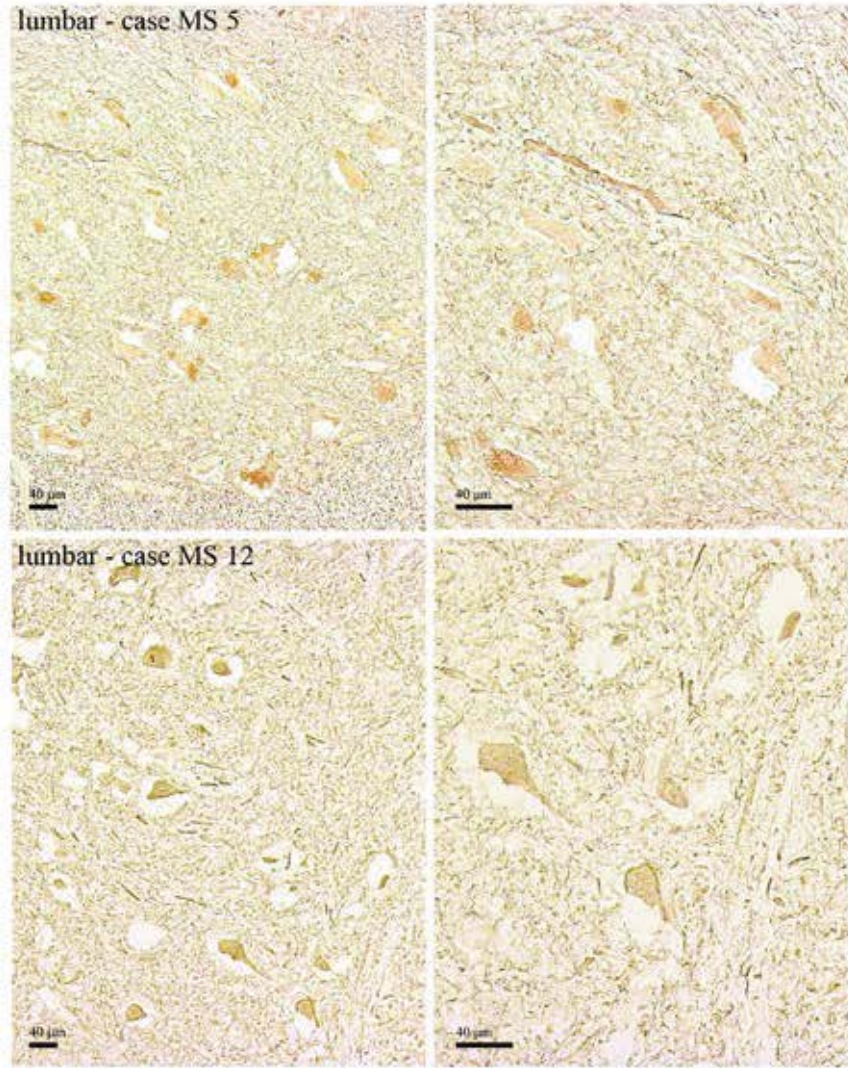
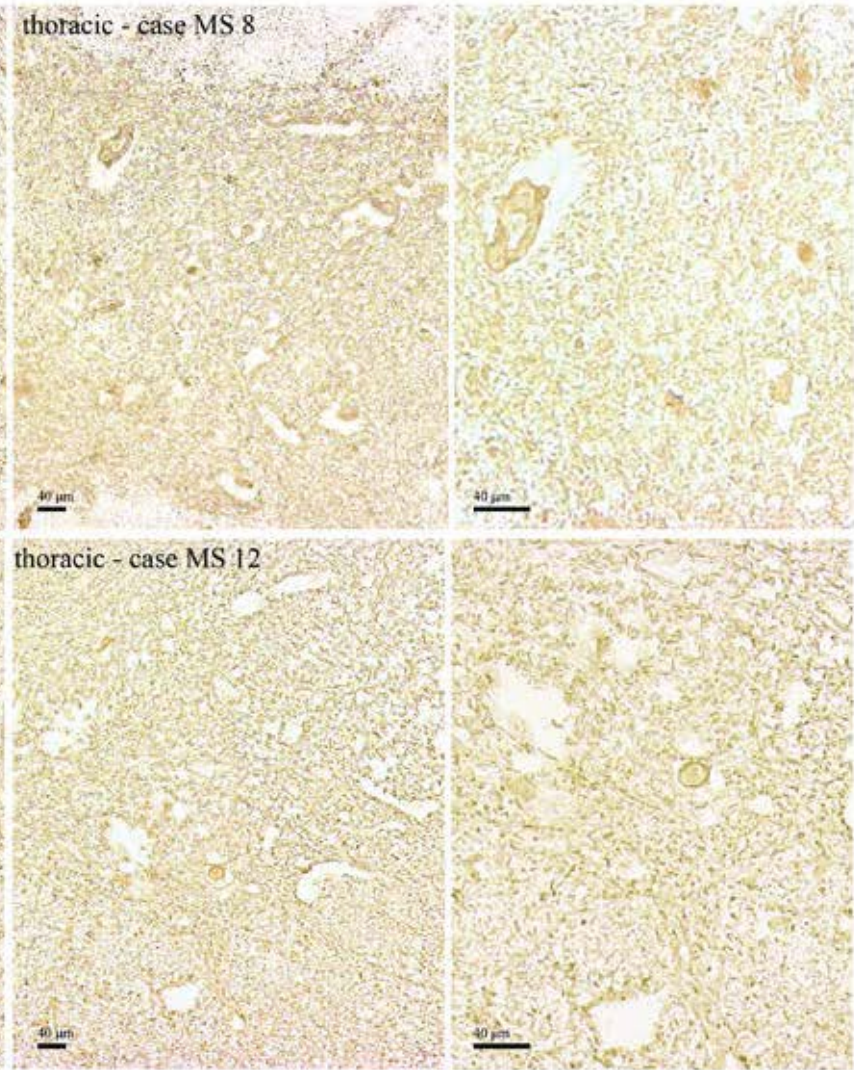

Figure 3: Perikarya expressing only abnormal, phosphorylated neurofilament H (P-NF-H) on lumbar (left column) and thoracic (right column) levels. The upper row shows two different patients, both with chronic silent lesions; the lower row shows differences within the same patient (also chronic silent lesions). Note the difference in intensity of the immunoreaction suggesting a higher amount of abnormal phosphorylated NF-H in distal levels of spinal cord. 


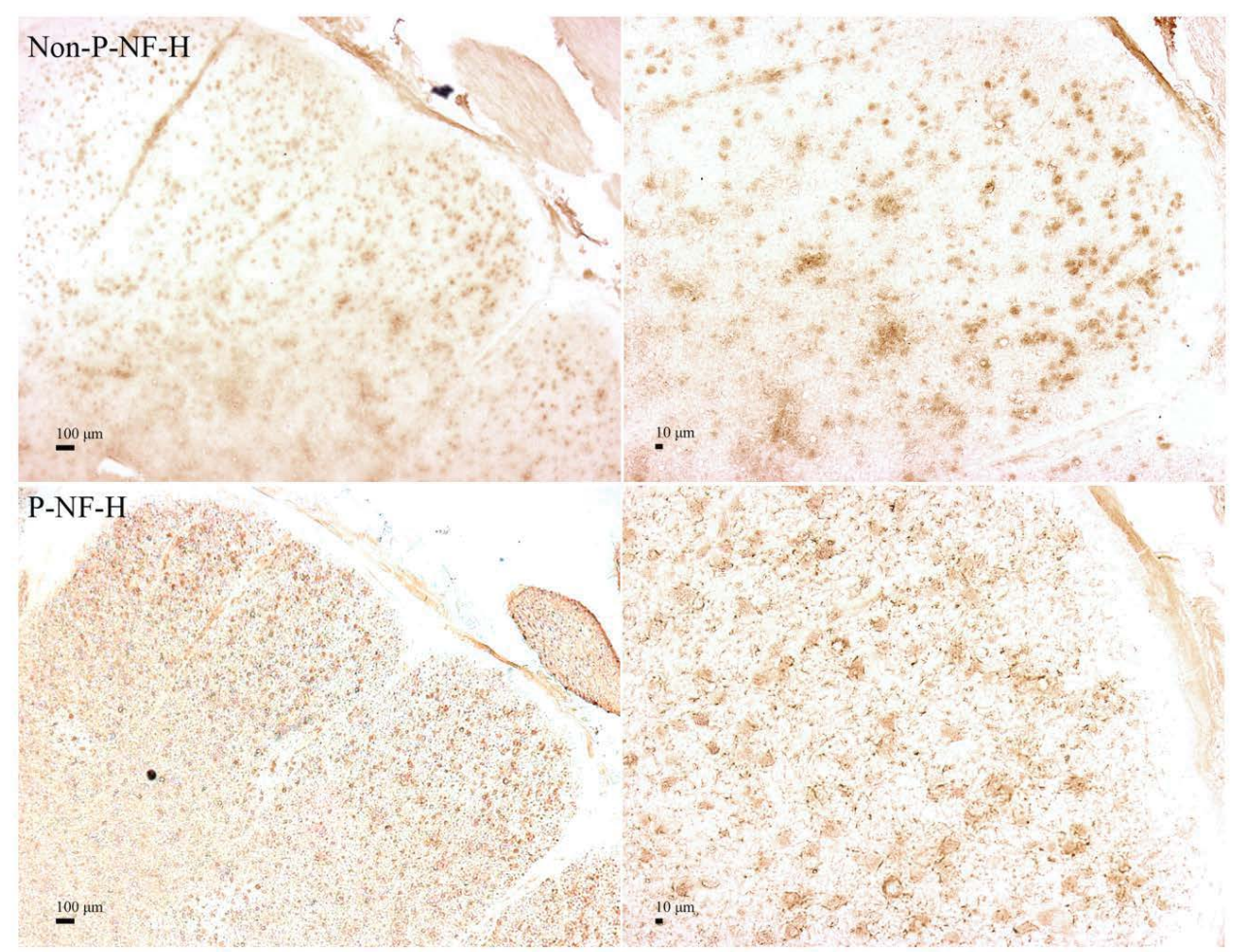

Figure 4: Swollen axons expressing non-phosphorylated neurofilament $\mathrm{H}$ (Non-P-NF-H, upper row) and phosphorylated neurofilament $\mathrm{H}$ (P-NF-H, lower row) in the dorsal horns of the spinal cord in a case with an acute MS lesion. Remarkable is the high expression of non-phosphorylated NF-H within axons, which, in this location (in contrast to the situation in perikarya), indicates pathology.

lesions. Schirmer et al. also reported a number of phosphorylated NF-H positive neurons in anterior horn cells of MS patients and noted that they were more pronounced in early and active lesions [12]. Considering the significant depletion of spinal motor neurons in MS that has been described in previous studies [10-12], it is likely that in non-immunoreactive areas of spinal cord, most damaged neurons have disappeared, although some may also have recovered. This hypothesis is supported by our comparison of the motor neuron count in a lumbar subgroup of 4 MS cases. 3 cases showing abnormal NF-H phosphorylation in perikarya (positive) had a notably higher motor neuron count than a single case that did not show abnormal NF-H phosphorylation (negative). Keeping in mind the low number of cases, this finding of course needs to be interpreted carefully, but it may suggest that abnormal NF phosphorylation indicates a damage neurons cannot survive on the long run. Schirmer et al. [12] describe upregulation of c-Jun in anterior horn neurons in or next to active and early inactive lesions, which may lead to cell death. Further studies are needed to explore whether the neurons with abnormally phosphorylated NF-H are indeed on the death pathway.

Mechanisms underlying these neuronal changes are currently under debate. Schirmer et al. attributed the damage and loss of neuronal cell bodies mainly to retrograde degeneration and stress reactions related to inflammation, demyelination and axonal damage in close proximity to perikarya [12]. In our chronic active MS cases, there was a correlation between inflammation and neurofilament immunopathology, but whether this inflammatory environment may have caused retrograde degeneration in the neurons cannot be stated with certainty. A decrease in synaptic input due to demyelination and/or damage to afferent axons was offered as an explanation for the neuronal apoptosis seen in cortical lesions [6] and this factor could also be causally related to damage to and loss of spinal motor neurons. Assuming that afferent fiber length (and therefore probability of lesions somewhere along the route) correlates with extent of anterior horn cell damage at respective levels, one might account for the greatest predisposition for damage occurring in lumbar samples compared to samples from more proximal levels.

The scientific application of the neurofilament profile is not limited to post-mortem neuropathologic studies in MS research, inasmuch as soluble NF levels in the cerebrospinal fluid (CSF) have been shown to be useful as biomarkers. NF-L levels have been found to reflect acute axonal damage and NF-H chronic irreversible damage, therefore implying prognostic value for disease progression or disability [23] In addition, immunosuppression therapies using Natalizumab or Fingolimod have been evaluated using soluble NF-L and NF-H levels in CSF with interesting results [24-26]. Thus, NF changes may become a useful measure in clinical trials and therapy in MS.

\section{Conclusion}

The present findings further implicate involvement of anterior horn cell perikarya in MS pathology as a common phenomenon. We propose that this occurs as a consequence of axonal damage to afferent and/or efferent fibres at early stages of lesion development and ultimately leads to substantial motor neuron loss in the spinal cord that may contribute to the irreversible disability common to chronic stages of the disease. Clearly, there is need for additional research in this field to elucidate further the mechanisms underlying this previously de-emphasized feature. The clinical and therapeutic implications of the phenomenon also remain subjects for future study. 


\section{Funding}

This work was supported by the Biomedical Sciences Exchange Program of the International Academy of Life Sciences, Bad Oeynhausen, Germany and by the Evangelisches Studienwerk Villigst, Germany (KMW); the United States Public Health Service (grants NS 08952 and NS 11920) (CSR); and the National MS Society (grant RG 1001-K-11) (CSR). CSR was the Wollowick Family Professor of Multiple Sclerosis Research at this Institution.

None of the sponsors had any influence in the study design, in the collection, analysis and interpretation of data, in the writing of the report nor in the decision to submit the article for publication.

\section{Acknowledgement}

The authors thank Miriam Pakingan for expert technical assistance; Kakuri Omari, PhD, for help with the immunohistochemistry; and Patricia Cobban-Bond for manuscript preparation.

\section{References}

1. Dutta R, Trapp BD (2007) Pathogenesis of axonal and neuronal damage in multiple sclerosis. Neurology 68: S22-31.

2. Raine CS, Cross AH (1989) Axonal dystrophy as a consequence of long-term demyelination. Lab Invest 60: 714-725.

3. Ferguson B, Matyszak MK, Esiri MM, Perry VH (1997) Axonal damage in acute multiple sclerosis lesions. Brain 120: 393-399.

4. Trapp BD, Peterson J, Ransohoff RM, Rudick R, Mörk S, et al. (1998) Axonal transection in the lesions of multiple sclerosis. N Engl J Med 338: 278-285.

5. Bitsch A, Schuchardt J, Bunkowski S, Kuhlmann T, Bruck W (2000) Acute axonal injury in multiple sclerosis. Correlation with demyelination and inflammation. Brain 123: 1174-1183.

6. Peterson JW, Bo L, Mork S, Chang A, Trapp BD (2001) Transected neurites, apoptotic neurons and reduced inflammation in cortical multiple sclerosis lesions. Ann neurol 50: 389-400.

7. Aktas O, Smorodchenko A, Brocke S, Infante-Duarte C, Schulze Topphoff U, et al. (2005) Neuronal damage in autoimmune neuroinflammation mediated by the death ligand TRAIL. Neuron 46: 421-432.

8. Vercellino M, Plano F, Votta B, Mutani R, Giordana MT, et al. (2005) Grey matter pathology in multiple sclerosis. Lancet Neurol 64: 1101-1107.

9. Smith T, Groom A, Zhu B, Turski L (2000) Autoimmune encephalomyelitis ameliorated by AMPA antagonists. Nat Med 6: 62-66.

10. Gilmore CP, DeLuca GC, Bo L, Owens T, Lowe J, et al. (2009) Spinal cord neuronal pathology in multiple sclerosis. Brain Pathol 19: 642-649.

11. Vogt J, Paul F, Aktas O, Muller-Wielsch K, Dorr J, et al. (2009) Lower motor neuron loss in multiple sclerosis and experimental autoimmune encephalomyelitis. Ann neurol 66: 310-322.
12. Schirmer L, Albert M, Buss A, Schulz-Schaeffer WJ, Antel JP et al. (2009) Substantial early, but non-progressive neuronal loss in multiple sclerosis (MS) spinal cord. Ann Neurol 66: 698-704.

13. Schlaepfer WW (1987) Neurofilaments: Structure, metabolism and implications in disease. J Neuropathol Exp Neurol 46: 117-129.

14. Sternberger LA, Sternberger NH (1983) Monoclonal antibodies distinguish phosphorylated and nonphosphorylated forms of neurofilaments in situ. Proc Natl Acad Sci U S A 80: 6126-6130.

15. Giasson BI, Mushynski WE (1996) Aberrant stress-induced phosphorylation of perikaryal neurofilaments. J Biol Chem 271: 30404-30409.

16. Gotow T (2000) Neurofilaments in health and disease. Medical Electron Microscopy: Official Journal of the Clinical Electron Microscopy Society of Japan 33: 173-199.

17. Bannerman PG, Hahn A, Ramirez S, Morley M, Bonnemann C, et al. (2005) Motor neuron pathology in experimental autoimmune encephalomyelitis: Studies in THY1-YFP transgenic mice. Brain 128: 1877-1886.

18. Bannerman PG, Hahn A (2007) Enhanced visualization of axonopathy in EAE using thy1-YFP transgenic mice. J Neurol Sci 260: 23-32.

19. Schirmer L, Antel JP, Bruck W, Stadelmann C (2011) Axonal loss and neurofilament phosphorylation changes accompany lesion development and clinical progression in multiple sclerosis. Brain Pathol 21: 428-440.

20. Raine CS (1997) The neuropathology of multiple sclerosis. In: Multiple sclerosis Clinical and pathogenetic basis; Raine CS, McFarland HF, Tourtellotte WW (eds). Chapman and Hall, London 151-171.

21. Kawamura Y, O'Brien P, Okazaki H, Dyck PJ (1977) Lumbar motoneurons of man II: The number and diameter distribution of large- and intermediatediameter cytons in "motoneuron columns" of spinal cord of man. J Neuropatho Exp Neurol 36: 861-870.

22. Mendonca DM, Chimelli L, Martinez AM (2005) Quantitative evidence fo neurofilament heavy subunit aggregation in motor neurons of spinal cords of patients with amyotrophic lateral sclerosis. Braz J Med Biol Res 38: 925-933.

23. Teunissen CE, Khalil M (2012) Neurofilaments as biomarkers in multiple sclerosis. Mult Scler 18: 552-556.

24. Gunnarsson $M$, Malmeström $C$, Axelsson $M$, Sundström $P$, Dahle $C$, et al. (2011) Axonal damage in relapsing multiple sclerosis is markedly reduced by natalizumab. Ann Neurol 69: 83-89.

25. Kuhle J, Malmeström C, Axelsson M, Plattner K, Yaldizli O, et al. (2013) Neurofilament light and heavy subunits compared as therapeutic biomarkers in multiple sclerosis. Acta Neurol Scand 128: e33-36.

26. Novakova L, Axelsson M, Khademi M, Zetterberg H, Blennow K, et al. (2017) Cerebrospinal fluid biomarkers of inflammation and degeneration as measures of fingolimod efficacy in multiple sclerosis. Mult Scler 23: 62-71.
Citation: Muller-Wielsch KS, Cannella B, Raine CS (2017) Multiple Sclerosis: Neurofilament Pathology in Spinal Motor Neurons. J Mult Scler (Foster City) 4 207. doi: 10.4172/2376-0389.1000207
OMICS International: Open Access Publication Benefits \&

\section{Features}

Unique features:

- Increased global visibility of articles through worldwide distribution and indexing

- Showcasing recent research output in a timely and updated manner

Special issues on the current trends of scientific research

Special features:

$700+$ Open Access Journals

$50,000+$ editorial team

Rapid review process

Quality and quick editorial, review and publication processing

Indexing at major indexing services

Sharing Option: Social Networking Enabled

- Authors, Reviewers and Editors rewarded with online Scientific Credits

- Better discount for your subsequent articles

Submit your manuscript at: http://www.omicsonline.org/submission/ 